\title{
Perceived Risk, Shared Benefit and Social Interaction in Vaccination
}

\section{By Alan McGreevy}

The spread of H1N1 during 2009 was stemmed, at least in part, by widespread voluntary vaccination. ${ }^{1}$ Every adult in Canada had the opportunity to consider the perceived costs and benefits of being vaccinated. What were perceived as the risks of being or not being vaccinated and what factors may have affected these perceptions? This paper will look at modeling vaccination response, taking into consideration the communication of risks and benefits.

The decision to not vaccinate is poorly understood. $^{2} \quad$ A case study found that only $22 \%$ of the staff at a Canadian health care centre received flu vaccines four or more times in the previous five years. ${ }^{3}$ This cannot be due to lack of access or information or a rejection of western medicine, and yet, for these people, the risks obviously outweighed the benefits. Being aware of rare and severe reactions to vaccines can have a significant impact on one's choice to vaccinate. ${ }^{4}$ An individual's perception of risk is based on experience and knowledge; events that are easily remembered or imagined are most significant in decision-making. ${ }^{5}$

Game theory compares courses of action based on risk - in this case, to be vaccinated or not. The accuracy of game theory relies on accurate models, advanced by the recent recognition that humans are, socially, best described as a scale-free network. ${ }^{6}$ This has been found with population dynamics tracked through sexual contact or transmission of avian influenza. ${ }^{7,8}$ This model reflects that we are more likely to be infected by immediate friends and neighbours and that some people have more neighbours than others. ${ }^{6}$ Risks change for those with many neighbours; they are more likely to have an infectious neighbour and, once infected, they are more likely to transmit the infection. ${ }^{6}$ These individuals have more to gain, personally and altruistically, by being vaccinated. The greater efficacy per vaccination makes these individuals critical in the efforts to control the spread of infectious disease. ${ }^{7-9}$ Reminders of this larger network and its interconnectivity can increase cognisance of the benefits of vaccination.

A survey by RAND Health in November 2009 regarding the uptake of seasonal influenza vaccine in the United States looked at the choice to vaccinate based on sources of flu-related information. ${ }^{10}$ Only $1 \%$ of respondents chose the H1N1 vaccine solely, suggesting that those who chose not to get the seasonal vaccine made a similar decision regarding the pandemic $\mathrm{H} 1 \mathrm{~N} 1$ vaccine. As predicted by a risk-perception model, there is a correlation between those who get flu-related information in more heterogeneous environments, where they are more aware of potentially infectious neighbours, and uptake of vaccine. Employers and healthcare providers were most effective as primary sources of information, increasing vaccination by four times and two times respectively. News media received in more isolated environments, was correlated with a decrease in vaccination. ${ }^{10}$ From this, it appears that $2009 \mathrm{H} 1 \mathrm{~N} 1$ information in media and advertising was plentiful but not persuasive.

There is evidence that the perception of risk can also apply to the shared benefit of vaccination: herd immunity. A population with a large percentage of individuals immune to infection has less chance of a chain of infection leading to susceptible or immunocompromised individuals; many vaccinations are more beneficial to society as a whole rather than the individual recipient. ${ }^{11}$ The RAND Health survey found that healthy adults who are in contact with, or caregivers of, higher risk individuals are significantly more likely to be vaccinated $(35 \%)$ than other healthy adults $(18 \%){ }^{10}$ However, in 2009, this group was included in the higher risk group, and was the least vaccinated higher risk population. The three most common reasons not to be vaccinated, accounting for $60 \%$ of 
responses, were: "I don't need it", "I don't believe in flu vaccines" and "I might get sick or experience side effects". ${ }^{10}$ Public health campaigns focusing on the herd immunity and diverse groups sharing contact enables the individual to take ownership of vaccination as a selfless act, stopping the spread of infection from reaching more vulnerable members of the community. ${ }^{12}$

When considering campaigns or resource distribution, governments and medical professionals should consider how individuals get vaccination information, and what affects their decisions. While news media may offer commentary on vaccination, persuasive power lies in the impact of human experience and social responsibility.

\section{Acknowledgements}

I would like to thank my colleagues at the National Centre for Foreign Animal Disease, particularly Yohannes Berhane, my advisor Hana Weingartl for her support, and Christina Fawcett for her proofreading.

\section{References}

1. Tosh PK, Jacobson RM, Poland GA. Influenza vaccines: from surveillance through production to protection. Mayo Clin Proc 2010;85(3):257-273.

2. Suk JE. Vaccine Safety: Misniformed about the Misinformed. Lancet Infect Dis 2010;10:144.

3. Mah M, Hagen N, Pauling-Shepherd K, Hawthorne J, Mysak M \& Louie, T. Understanding influenza vaccination attitudes at a Canadian cancer center. Am J Infect Control 2005;33(4):243-250.

4. The Lancet Infectious Diseases. Vaccine Safety: informing the misinformed. Lancet Infect Dis 2009;9:719.

5. Tversky A, Kahneman D. Availability: a heuristic for judging frequency and probability. Cognitive Psychology 1973;5:207-232.

6. Zhang, H, Zhang, J, Zhou, C, Small, M \& Wang, B. Hub nodes inhibit the outbreak of epidemic under voluntary vaccination. New J Phys 2010;12:023015.

7. Liljeros, F, Edling, CR, Nunes Amaral, LA, Stanley, HE $\&$ Berg, Y. The web of human sexual contacts. Nature 2001;411:907-908.

8. Small, M, Walker, DM \& Tse, CK. The Distribution of Avian Influenza Outbreaks. Phys Rev Lett 2007;99(18):188702.

9. Loeb M, Russel ML, Moss L, Fonesca K, Fox J, Earn DJD, Aoki F, Horsman G, Van Caeseele P, Chokani K, Vooght M, Babiuk L, Webby R, Walter SD. Effects of Influenza Vaccination of Children on Infection Rates in Hutterite Communities: A Randomized Trial. JAMA 2010;303(10):943-950.

10. Harris, K, Maurer, J \& Usher-Pines, L. Seasonal Influenza Vaccine Use by Adults in the U.S. RAND Health 2009;OP289.
11. Fine PE, Clarkson AJ. Individual versus public priorities in the determination of optimal vaccination policies. Am J Epidemiol 1986; 124(6):1012-20.

12. Moyad MA. Conventional and alternative medical advice for cold and flu prevention: what should be recommended and what should be avoided? Urol Nurs 2009;29(5):455458.

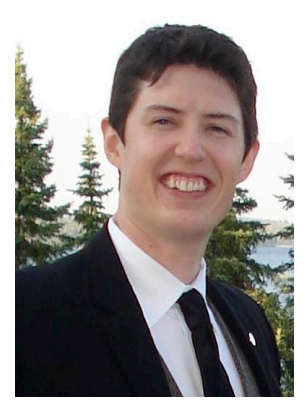

\section{Author Profile}

Alan McGreevy is a MSc candidate studying Rift Valley fever virus at the University of Manitoba (Department of Medical Microbiology). His research interests include high-containment viral pathogens, biosafety and counter-terrorism, and evolutionary genetics. 